\title{
CESÀRO KERNELS ON CLASSICAL GROUPS
}

\author{
DASHAN FAN
}

(Received 6 June 1996; revised 18 October 1996)

Communicated by A. H. Dooley

\begin{abstract}
We study the Cesàro operator $K_{N}^{\alpha} * f(U)$ on the classical group $G$ and give a necessary and sufficient condition on the index $\alpha=\alpha(G)$ for which the operator $K_{N}^{\alpha} * f(U)$ is convergent to $f(U)$ for any continuous function $f$ as $N \rightarrow \infty$. The result in this paper solves a question posed by Gong in the book Harmonic analysis on classical groups.
\end{abstract}

1994 Mathematics subject classification (Amer. Math. Soc.): primary 43A55, 43A50, 43A80; secondary 22E20.

Keywords and phrases: Fourier series, Cesàro means, classical group, Lebesgue constant.

\section{Introduction}

Let $G$ be a connected, simply connected, compact Lie group of dimension $k$. Let $\mathfrak{g}$ be the Lie algebra of $G$ and $t$ the Lie algebra of a fixed maximal torus $\pi$ in $G$ of dimension $l$. Let $A$ be a system of positive roots for $(\mathfrak{g}, t)$, and let $\beta=2^{-1} \sum_{\alpha \in A} \alpha$.

Let $|$.$| be the norm on \mathfrak{g}$ induced by the negative of the Killing form $B$ on $\mathfrak{g}^{\mathbb{C}}$, the complexification of $\mathfrak{g}$; then $|$.$| induces a bi-invariant metric d$ on $G$. Furthermore, since $\left.B\right|_{t^{\mathrm{C}} \times t^{\mathrm{C}}}$ is non-degenerate, given $\lambda \in \operatorname{hom}_{\mathbb{C}}\left(t^{\mathbb{C}}, \mathbb{C}\right)$ there is a unique $H_{\lambda}$ in $t^{\mathbb{C}}$ such that $\lambda(H)=B\left(H, H_{\lambda}\right)$ for each $H \in t^{\mathbb{C}}$. We let $\langle$,$\rangle and \|\|$ denote the inner product and norm transferred from $t$ to $t^{*}$ (the dual of $t$ ) by means of this canonical isomorphism.

Let $\mathbb{N}=\{H \in t, \exp H=I\}$, where $I$ is the identity in $G$. The weight lattice $P$ is defined by $P=\{\lambda \in t,\langle\lambda, x\rangle \in 2 \pi \mathbb{Z}$, any $x \in \mathbb{N}\}$ with dominant weights defined by $\Lambda=\{\lambda \in P,\langle\lambda, \alpha\rangle \geq 0$ any $\alpha \in A\}$. We can identify $\hat{G}$ with this $\Lambda$ because $\Lambda$ provides a full set of parameters for the equivalence classes of unitary irreducible

This research was partially supported by a grant of The Graduate School Research Committee of the University of Wisconsin-Milwaukee.

(C) 1997 Australian Mathematical Society $0263-6115 / 97 \$ A 2.00+0.00$ 
representations of $G$. For $\lambda \in \Lambda$, the representation $U_{\lambda}$ has dimension

$$
d_{\lambda}=\prod_{\alpha \in A} \frac{\langle\lambda+\beta, \alpha\rangle}{\langle\beta, \alpha\rangle}
$$

and its associated character is

$$
\chi_{\lambda}(x)=\frac{\sum_{w \in W} \epsilon(w) e^{i\langle w(\lambda+\beta), x\rangle}}{\sum_{w \in W} \epsilon(w) e^{i(w \beta \cdot x\rangle}}
$$

where $x \in t, W$ is the Weyl group and $\epsilon(w)$ is the signature of $w \in W$. For any Lebesgue integrable function $f$ on $G, f$ has the Fourier expansion

$$
f(x) \sim \sum_{\lambda \in \Lambda} d_{\lambda} \chi_{\lambda} * f(x) .
$$

In this paper our particular interest is on the classical compact Lie groups: rotation group $\mathrm{SO}(n)$, unitary group $\mathrm{U}(n)$ and unitary symplectic group $\operatorname{USP}(2 n)$, since these three groups are the Shilov boundaries of certain bounded symmetric domains in several complex variables. More specifically, $\mathrm{SO}(n)$ is the Shilov boundary of the real classical domain of type one; $\mathrm{U}(n)$ and $\operatorname{USP}(2 n)$ are the Shilov boundaries of the complex classical domain of type one and the classical domain of quaternions of type one, respectively. Readers can see $[\mathrm{C}]$ and $[\mathrm{H}]$ for more details of how these domains play important roles in the study of several complex variables.

Let $\Omega$ be either the real or the complex classical domain of type one. Hua calculated the automorphism group $\operatorname{Aut}(\Omega)$ and used its Jacobian determinant to define the Poisson kernel $P(X, \Gamma)$ so that he was able to solve the Dirichlet problem on $\Omega$ (see $[\mathrm{H}]$ ).

After finding explicit formulas for the Poisson kernels, Gong defined the Cesàro kernels $K_{N}^{\alpha}$ on the classical groups (see 1.1 in the next section and 6.1, 6.3 in Section 6). An important feature of Gong's Cesàro kernels is that if one chooses $\alpha=\alpha(G, N)$ to be a certain sequence going to infinity then the Cesàro kernels will coincide with the Poisson kernels in a certain sense, which enables one to find the Poisson kernel on USP $(2 n)$ and solve the Dirichlet problem on the classical domain $\Omega$ of quaternions where the automorphism group $\operatorname{Aut}(\Omega)$ is difficult to calculate. These details can be found in Section 11.6 of [G].

Let $d V$ be the normalized Haar measure on $G$. The critical index $\alpha=\alpha(G)$ of the Cesàro kernel on $G$ is defined by $\alpha(G)=(n-2) /(n-1)$ if $G=\operatorname{SO}(n)$, $\alpha(G)=(n-1) / n$ if $G=\mathrm{U}(n)$ and $\alpha(G)=(2 n-2) /(2 n+1)$ if $G=\operatorname{USP}(2 n)$.

For $\alpha>-1$, let

$$
B_{N, \lambda}^{\alpha}=d_{\lambda}^{-1} \int_{G} \chi_{\lambda}\left(V^{-1}\right) K_{N}^{\alpha}(V) d V
$$

and define the Cesàro $(C, \alpha)$ mean of the Fourier series for a function $f$ by

$$
\sum_{\lambda \in \Lambda} B_{N . \lambda}^{\alpha} d_{\lambda} \chi_{\lambda} * f
$$


It is well known that $\mathrm{SO}(2)=\mathrm{U}(1)$ is the one dimensional torus $\mathbb{T}^{1}$; from [G] one easily sees that when $G=\mathrm{SO}(2)=\mathrm{U}(1)$ the above definition is the classical $(C, \alpha)$ mean of the Fourier series on $\mathbb{T}^{1}$. Also from $[G]$, one knows that for a Lebesgue integrable function $f$

$$
\sum_{\lambda \in \Lambda} B_{N, \lambda}^{\alpha} d_{\lambda} \chi_{\lambda} * f(U)=\int_{G} K_{N}^{\alpha}(V U) f(V) d V=\left(K_{N}^{\alpha} * f\right)(U)
$$

The following convergence theorem about this Cesàro operator $K_{N}^{\alpha} * f$ can be found in [G, Chapters 2, 7 and 11].

THEOREM A. Suppose that $f$ is a continuous function on $G$. Then $\lim _{N \rightarrow \infty}\left(K_{N}^{\alpha} *\right.$ $f)(U)=f(U)$ for each $U \in G$, provided $\alpha>\alpha(G)$.

The condition $\alpha>\alpha(G)$ in the above theorem is sharp when $G=\mathrm{SO}(2)$ (see [Z]), so Gong asked in [G] whether the condition $\alpha>\alpha(G)$ can be further improved when $\operatorname{dim}(G)>1$.

The main purpose of this paper is to solve the above question. Readers will see that the interesting fact is that on $\operatorname{SO}(n)$ the condition $\alpha>\alpha(G)$ can be improved to the sharp condition $\alpha \geq \alpha(G)$ only if $n$ is odd; on $\mathrm{U}(n)$ the condition $\alpha>\alpha(G)$ can be improved to the sharp condition $\alpha \geq \alpha(G)$ only if $n$ is even, and on $\operatorname{USP}(2 n)$ the condition $\alpha>\alpha(G)$ is sharp for any $n$.

In this paper, we are not able to give a unified proof for the above results on different groups due to some slight differences among the Cesàso kernels and among the Weyl functions. But the proofs on these three groups are essentially the same with minor modifications only on some elementary computations. For this reason, we will give the detail only on $\operatorname{SO}(n)$, and list the theorems on $\mathrm{U}(n)$ and $\operatorname{USP}(2 n)$ in Section 6 without giving proofs.

As readers shall see the definitions of the Cesàro kernels, it is natural to define an analogue of the Cesàro kernel on a general compact Lie group and study its convergence properties. The possibility of such an extension will be discussed in the last section. In contrast with this Cesàro kernel defined via the 'kernel side', on a general compact Lie group one usually studies certain summability kernels via the Fourier series side. For instance, one can consider suitable functions $\Phi_{N}$ on $\Lambda$ such that $\lim _{N \rightarrow \infty} \Phi_{N}(\lambda)=1$ for each $\lambda \in \Lambda$. Then one defines a summability kernel $K_{N}^{\Phi}=\sum_{\lambda \in \Lambda} \Phi_{N}(\lambda) d_{\lambda} \chi_{\lambda}$ and studies the convergence property for $\lim _{N \rightarrow \infty} K_{N}^{\Phi} * f$. Readers may refer [Cl, M, GST1, GST2 and F2] for further information about various summability kernels on a general compact Lie group. 


\section{Cesàro Kernels on $\mathrm{SO}(n)$}

For $\alpha>-1$ and a positive integer $N$, let $A_{N}^{\alpha}=(\alpha+N) \cdots(\alpha+1) / N$ !. Then the Cesàro kernel $K_{N}^{\alpha}(V)$ on $\mathrm{SO}(n)$ is defined by

$$
K_{N}^{\alpha}(V)=\operatorname{det}^{(n-1) / 2}\left(\left\{A_{N}^{\alpha} I+\sum_{j=1}^{N}\left(V^{j}+V^{\prime j}\right) \sum_{\nu=0}^{N-j} A_{v}^{\alpha-1}\right\} / A_{N}^{\alpha}\right) / B_{n}^{\alpha},
$$

where

$$
B_{N}^{\alpha}=\int_{\mathrm{SO}(n)} \operatorname{det}^{(n-1) / 2}\left(\left\{A_{N}^{\alpha} I+\sum_{j=1}^{N}\left(V^{j}+V^{j}\right) \sum_{v=0}^{N-j} A_{v}^{\alpha-1}\right\} / A_{N}^{\alpha}\right) d V
$$

and $V^{\prime}$ is the transpose of the matrix $V$. One easily sees, from the above definition, that for any integer $N$,

$$
\int_{\mathrm{SO}(n)} K_{N}^{\alpha}(U) d U=1
$$

Recall that any $V \in \mathrm{SO}(2 k)$ is conjugate to an element $\exp \theta$ in the maximal torus $\mathrm{T}$. Let $\theta=\left(\theta_{1} \ldots \theta_{l}\right)$ be the regular coordinate and let $\sigma_{N}^{\alpha}(t)=1 / 2+$ $\sum_{j=1}^{N} \cos j t A_{N-j}^{\alpha} / A_{N}^{\alpha}$ be the one-dimensional Cesàro kernel (see [Z]). Then from [F1], we know that the Cesàro kernel can be written by the following manageable form

$$
K_{N}^{\alpha}(V)=\prod_{j=1}^{l}\left\{\sigma_{N}^{\alpha}\left(\theta_{j}\right)\right\}^{n-1} / \tilde{B}_{N}^{\alpha},
$$

where

$$
\tilde{B}_{N}^{\alpha}=\int_{\mathrm{SO}(n)} \prod_{j=1}^{l}\left\{\sigma_{N}^{\alpha}\left(\theta_{j}\right)\right\}^{n-1} d V
$$

Let $\alpha_{0}=(n-2) /(n-1)$ be the critical index for the Cesàro mean on $\operatorname{SO}(n)$. We will prove that if $n=2 k+1$ then $K_{N}^{\alpha} * f(U)$ converges to $f(U)$ for any continuous function $f$ if and only if $\alpha \geq \alpha_{0}$; if $n=2 k$ then $K_{N}^{\alpha} * f(U)$ converges to $f(U)$ for any continuous function $f$ if and only if $\alpha>\alpha_{0}$.

To prove the case of $n=2 k+1$ and $\alpha=\alpha_{0}$, we notice that the kernel $K_{N}^{\alpha_{0}}(U)$ in this case is positive so that we first prove a lemma on any compact Lie group $G$ which states that if for each positive integer $N, T_{N}(f)(U)=\int_{G} f(V U) K_{N}(V) d V$ is a positive operator and $T_{N}(1)=1$ then as $N \rightarrow \infty, T_{N}(f)(U)$ converges to $f(U)$ for any continuous function $f$ if and only if $\lim _{N \rightarrow \infty} \int_{G} d(V, I)^{2} K_{N}(V) d V=$ 0 , where $d(V, I)$ is the distance between $V$ and $I$. Next we write the integral $\int_{\mathrm{SO}(n)} d(V, I)^{2} K_{N}^{\alpha_{0}}(V) d V=\tilde{J}_{N}^{\alpha_{0}} / J_{N}^{\alpha_{0}}$. Thus the problem is reduced to proving that 
$\tilde{J}_{N}^{\alpha_{0}}=O\left(N^{-k}\right)$ and $J_{N}^{\alpha_{0}} \geq A N^{-k} \log N$ as $N \rightarrow \infty$, where $A$ is a positive constant independent of $N$. We obtain that result after dividing the integral into several pieces and checking that each piece is under control. Some of this checking requires a careful tracking of the exponents of $N$ in a large number of cases. In the case $n=2 k+1$ and $\alpha<\alpha_{0}$, the function $g(V)=1-\cos \theta_{1}$, with $\exp \theta$ being a conjugate matrix of $V$, is proved to be a counter example showing that the convergence theorem fails. For the case $n=2 k$ and $\alpha \leq \alpha_{0}$, we calculate the Lebesgue constants and show that all of them are unbounded. The Banach-Steinhaus theorem then gives the divergence conclusion.

Now we state our main results on $\mathrm{SO}(n)$ in the following theorems.

THEOREM 1. Suppose that $f(U)$ is a continuous function on $\operatorname{SO}(2 k+1)$. Then

$$
\lim _{N \rightarrow \infty} \int_{\mathrm{SO}(2 k+1)} K_{N}^{\alpha_{0}}(V U) f(V) d V=f(U) .
$$

THEOREM 2. There exists a $C^{\infty}$ function $g(U)$ on $\mathrm{SO}(2 k+1)$ such that

$$
\limsup _{N \rightarrow \infty} \int_{\mathrm{SO}(2 k+1)} K_{N}^{\alpha}(V) g(V) d V \neq g(I) \quad \text { for any } \quad-1<\alpha<\alpha_{0} .
$$

THEOREM 3. For sufficiently large $N>0$, we have

$$
\int_{\mathrm{SO}(2 k)}\left|K_{N}^{\alpha_{0}}(V)\right| d V \geq A \log N
$$

where $A$ is a positive constant independent of $N$.

THEOREM 4. For any $-1<\alpha<\alpha_{0}$ there exists an $\epsilon>0$ such that, for sufficiently large $N>0$,

$$
\int_{\mathrm{SO}(2 k)}\left|K_{N}^{\alpha}(V)\right| d V \geq A N^{\epsilon}
$$

where $A$ is a positive constant independent of $N$.

Clearly, Theorems 1 to 4 and Theorem A together with the Banach-Steinhaus theorem lead to the following theorem which solves the question of Gong on the rotation group $\mathrm{SO}(n)$.

THEOREM B. If $n$ is odd, then $\lim _{N \rightarrow \infty} K_{N}^{\alpha} * f(U)=f(U)$ for any continuous function $f$ on $\mathrm{SO}(n)$ if and only if $\alpha \geq(n-2) /(n-1)$.

If $n$ is even, then $\lim _{N \rightarrow \infty} K_{N}^{\alpha} * f(U)=f(U)$ for any continuous function $f$ on $\mathrm{SO}(n)$ if and only if $\alpha>(n-2) /(n-1)$. 
This paper is organized as follows. In the second section we will give some preliminary lemmas for proving the theorems. Theorem 1 will be proved in the third section. Theorem 3 and Theorem 4 will be proved in the fourth section and Theorem 2 will be proved in the fifth section. In the sixth section we will answer the same question on other two groups $\mathrm{U}(n)$ and $\mathrm{USP}(2 n)$. Finally in Section 7 , we will discuss the possible extension of the Cesàro kernels to a general compact Lie group.

Throughout this paper, the letter $A$ will denote a positive constant which may vary at each occurrence but independent of the essential variables or functions. In particular, this $A$ will be independent of the integer $N$. This independence will be clear from the context. Also we use $f_{N} \cong g_{N}$ to mean that there exist positive numbers $A, B$ and $C$ such that $A f_{N} \leq B g_{N} \leq C f_{N}$, where $A, B$ and $C$ are independent of the variable $N$.

\section{Some preliminary lemmas}

Let $K_{N}(V)$ be a non-negative function on $G$ for each integer $N$. The linear positive operator $T_{N}$ associated to this kernel $K_{N}$ is defined by

$$
T_{N}(f)(U)=\int_{G} f(V U) K_{N}(V) d V .
$$

For $N=1,2, \ldots$, considering the operator sequence $\left\{T_{N}\right\}$, we have the following lemma that is true on any compact Lie group $G$.

LEMMA 2.2. Let $d(V, I)$ be the distance between $I$ and $V \in G$. Suppose that $T_{N}(1)=1$ for all integers $N$. Then

$$
\lim _{N \rightarrow \infty} \int_{G} f(V U) K_{N}(V) d V=f(U)
$$

for any continuous function $f(U)$ if and only if

$$
\lim _{N \rightarrow \infty} \int_{G} d(V, I)^{2} K_{N}(V) d V=0 .
$$

PROOF. Let $M$ be a positive constant such that $\|f\|_{\infty} \leq M$. Now because $G$ is compact and $f$ is continuous, for any $\epsilon>0$ one can choose a $\delta>0$ such that $-\epsilon<f(V U)-f(U)<\epsilon$ if $d(V, I)<\delta$. Therefore for all $U$ and $V$ in $G$,

$$
-\epsilon-2 M \delta^{-2} d^{2}(V, I)<f(V U)-f(U)<\epsilon+2 M \delta^{-2} d^{2}(V, I) .
$$

Noting that $T_{N}$ is a positive operator with $T_{N}(1)=1$, we easily obtain that

$$
\begin{aligned}
-\epsilon-2 M \delta^{-2} \int_{G} d^{2}(V, I) K_{N}(V) d V & <\int_{G} f(V U) K_{N}(V) d V-f(U) \\
& <\epsilon+2 M \delta^{-2} \int_{G} d^{2}(V, I) K_{N}(V) d V .
\end{aligned}
$$


Therefore, (2.3) holds for any continuous function $f$ if and only if

$$
\lim _{N \rightarrow \infty} \int_{G} d^{2}(V, I) K_{N}(V) d V=0 .
$$

The lemma is proved.

We now recall the following estimates of the one dimensional Cesàro kernels $\sigma_{n}^{\alpha}(\theta)$.

LEMmA 2.8. If $\alpha>-1$ and $|\theta| \leq N^{-1}$, then $\left|\sigma_{N}^{\alpha}(\theta)\right| \cong N$. If $\alpha>-1$ and $|\theta|>N^{-1}$, then

$$
\begin{aligned}
\left\{\sigma_{N}^{\alpha}(\theta)\right\}^{n}= & \sin ^{n}\{(N+(1+\alpha) / 2) \theta-\pi \alpha / 2\} /\left\{(\alpha)_{N}^{n}(2 \sin (\theta / 2))^{n(\alpha+1)}\right\} \\
& +O\left(N^{-(n-1) \alpha-1} \theta^{-(n-1)(\alpha+1)-2}\right),
\end{aligned}
$$

where $(\alpha)_{N}=\Gamma(\alpha+N+1) /\{\Gamma(\alpha+1) \Gamma(N+1)\} \cong N^{\alpha}$ for $N$ sufficiently large. In particular, for $\alpha>-1$ and $|\theta|>N^{-1}$ one has

$$
\left\{\sigma_{N}^{\alpha}(\theta)\right\}^{n}=O\left(N^{-n \alpha}|\theta|^{-n(\alpha+1)}\right) .
$$

The proof of the lemma can be found in [Z, p. 77 and p. 95]. More precisely, (2.9) is an easy modification of (5.14) in [Z, p. 95].

LEMMA 2.10. Let $n=2 k+1$ and $\alpha_{0}=(n-2) /(n-1)=(2 k-1) /(2 k)$. Then, as $N \rightarrow \infty$,

$$
J^{\alpha_{0}}=\int_{-\pi}^{\pi} \cdots \int_{-\pi}^{\pi} \prod_{j=1}^{k}\left|\sigma_{N}^{\alpha_{0}}\left(\theta_{j}\right)\right|^{2 k} D(\theta) d \theta_{1} \cdots d \theta_{k} \geq A N^{-k} \log N
$$

where $D(\theta)=D\left(\theta_{1}, \ldots, \theta_{k}\right)=\prod_{i=1}^{k}(1-\cos \theta) \prod_{1 \leq i<j \leq k}\left(\cos \theta_{i}-\cos \theta_{j}\right)^{2}$ is the Weyl function on $\mathrm{SO}(2 k+1)$.

PROOF. The case $k=1$ is $[\mathrm{F} 1,(16)]$. For $k \geq 2$, we write

$$
D\left(\theta_{1}, \theta_{2}, \ldots, \theta_{k}\right)=\left(1-\cos \theta_{1}\right) \prod_{i=1}^{k}\left\{\left(1-\cos \theta_{i}\right)-\left(1-\cos \theta_{1}\right)\right\}^{2} D\left(\theta_{2}, \ldots, \theta_{k}\right) .
$$

Now we notice that for $\theta=\left(\theta_{1}, \theta_{2}, \ldots, \theta_{k}\right)$ with $\left|\theta_{j}\right|<1 / N, j=2,3, \ldots, k$ and $1 / N \leq \theta_{1}<\pi / 4$

$$
\begin{aligned}
D(\theta) & =\left(1-\cos \theta_{1}\right) \prod_{i=2}^{k}\left\{\left(1-\cos \theta_{1}\right)^{2}+O\left(N^{-2}\right)\left(1-\cos \theta_{1}\right)+O\left(N^{-4}\right)\right\} D\left(\theta_{2}, \ldots, \theta_{k}\right) \\
& =\left\{\left(1-\cos \theta_{1}\right)^{2 k-1}+\sum_{p=1}^{2 k-1}\left(1-\cos \theta_{1}\right)^{2 k-1-p} O\left(N^{-2 p}\right)\right\} D\left(\theta_{2}, \ldots, \theta_{k}\right) .
\end{aligned}
$$


We let

$$
J^{\alpha_{0}} \geq \int_{1 / N}^{\pi / 4} \int_{0}^{1 / N} \cdots \int_{0}^{1 / N} \prod_{j=1}^{k}\left|\sigma_{N}^{\alpha_{0}}\left(\theta_{j}\right)\right|^{2 k} D\left(\theta_{1}, \ldots, \theta_{k}\right) d \theta_{1} \cdots d \theta_{k}
$$

By Lemma 2.8 we easily see

$$
\begin{aligned}
J^{\alpha_{0}} \geq & A N^{2 k(k-1)-2 p} \int_{1 / N}^{\pi / 4}\left|\sigma_{N}^{\alpha_{0}}\left(\theta_{1}\right)\right|^{2 k}\left(1-\cos \theta_{1}\right)^{2 k-1} d \theta_{1}|B(I, 1 / N)| \\
& +O\left(\sum_{p=1}^{2 k-1} N^{2 k(k-1)-2 p} \int_{1 / N}^{\pi / 4}\left|\sigma_{N}^{\alpha_{0}}\left(\theta_{1}\right)\right|^{2 k}\left(1-\cos \theta_{1}\right)^{2 k-1-p} d \theta_{1}|B(I, 1 / N)|\right) \\
= & J_{1}+J_{2} .
\end{aligned}
$$

In the above estimate $B(I, 1 / N)$ is the ball in $\operatorname{SO}(2 k-1)$ with center $I$ and radius $1 / N$, and $|B(I, 1 / N)|, \cong \int_{-1 / N}^{1 / N} \cdots \int_{-1 / N}^{1 / N} D\left(\theta_{2}, \ldots, \theta_{k}\right) d \theta_{2} \cdots d \theta_{k}$, is the Haar measure of $B(I, 1 / N)$ so that $|B(I, 1 / N)| \cong N^{-\operatorname{dim}(\operatorname{SO}(2 k-1))}=N^{-(k-1)(2 k-1)}$. Thus using the estimate $(1-\cos \theta) \cong \theta^{2}$ we have

$$
\begin{aligned}
J_{1} & \geq A N^{2 k(k-1)-(2 k-1)(k-1)} \int_{0}^{\pi / 4}\left|\sigma_{N}^{\alpha_{0}}\left(\theta_{1}\right)\right|^{2 k} \theta_{1}^{4 k-2} d \theta_{1} \\
& =A N^{k-1} \int_{0}^{\pi / 4}\left|\sigma_{N}^{\alpha_{0}}\right|^{2 k} \theta^{4 k-2} d \theta
\end{aligned}
$$

By using formula (2.9), we now obtain that

$$
\begin{aligned}
J_{1} \geq & \left.A N^{-k} \int_{1 N}^{\pi / 4} \sin ^{2 k}\left(N+\left(\alpha_{0}+1\right) / 2\right) \theta-\alpha \pi / 2\right) / \theta d \theta \\
& +O\left(N^{-k-1 / 2 k} \int_{1 / N}^{\pi / 4} \theta^{-1-1 / 2 k} d \theta\right) \geq A N^{-k} \log N, \quad(N \rightarrow \infty) .
\end{aligned}
$$

Also by using the estimate $\sigma_{N}^{\alpha_{0}}(\theta)=O\left(N^{-\alpha_{0}}|\theta|^{-\alpha_{0}-1}\right)$ for $\alpha_{0}=(2 k-1) /(2 k)$, we see that

$$
\begin{aligned}
J_{2} & =O\left(\sum_{p=1}^{2 k-1} N^{k-1-2 p} \int_{0}^{\pi / 4} N^{-2 k+1} \theta^{-4 k+1} \theta^{4 k-2-2 p} d \theta\right) \\
& =O\left(\sum_{p=1}^{2 k-1} N^{-k-2 p} \int_{1 / N}^{\pi / 4} \theta^{-1-2 p} d \theta\right)=O\left(N^{-k}\right)
\end{aligned}
$$

Thus the proof of Lemma 2.10 is complete.

Similarly to Lemma 2.10 , we have the following lemma for $n=2 k$ : 
LEMMA $2.10^{\prime}$. Let $n=2 k$ and $\alpha_{0}=(n-2) /(n-1)=(2 k-1) /(2 k)$. Then

$$
\mathscr{J}^{\alpha_{0}}=\int_{-\pi}^{\pi} \cdots \int_{-\pi}^{\pi} \prod_{j=1}^{k}\left|\sigma_{N}^{\alpha_{0}}\left(\theta_{j}\right)\right|^{2 k-1} \Delta(\theta) d \theta_{1} \cdots d \theta_{k} \geq A \log N, \quad(N \rightarrow \infty)
$$

where $\Delta(\theta)=\Delta\left(\theta_{1}, \ldots, \theta_{k}\right)=\prod_{1 \leq i<j \leq k}\left(\cos \theta_{i}-\cos \theta_{j}\right)^{2}$ is the Weyl function on $\mathrm{SO}(2 k)$.

PROOF. We write

$$
\Delta\left(\theta_{1}, \theta_{2}, \ldots, \theta_{k}\right)=\prod_{j=2}^{k}\left(\left(1-\cos \theta_{j}\right)-\left(1-\cos \theta_{1}\right)\right)^{2} \Delta\left(\theta_{2}, \theta_{3}, \ldots, \theta_{k}\right) .
$$

Then we have

$$
\begin{gathered}
\mathscr{J}^{\alpha_{0}}=\int_{0}^{1 / N} \cdots \int_{0}^{1 / N}\left(\int_{1 / N}^{\pi / 4}\left|\sigma_{N}^{\alpha_{0}}\left(\theta_{1}\right)\right|^{2 k-1} \prod_{j=2}^{k}\left(\left(1-\cos \theta_{1}\right)-\left(1-\cos \theta_{j}\right)^{2}\right) d \theta_{1}\right) \\
\prod_{j=2}^{k}\left|\sigma_{N}^{\alpha_{0}}\left(\theta_{j}\right)\right|^{2 k-1} \Delta\left(\theta_{2}, \ldots, \theta_{k}\right) d \theta_{2} \cdots d \theta_{k} .
\end{gathered}
$$

Now using the same argument as in Lemma 2.10 we can easily obtain Lemma $2.10^{\prime}$.

NOTE. We obtain the estimate $\mathscr{J}^{\alpha_{0}} \geq A \log N$ in Lemma $2.10^{\prime}$ instead of the estimate $\mathscr{J}^{\alpha_{0}}>A N^{-k} \log N$ in Lemma 2.10 since the Weyl functions $\Delta(\theta)$ and $D(\theta)$ are different.

LEMMA 2.11. Suppose that $m$ is a positive integer and $\eta$ is any real number. Let

$$
I_{N}^{\alpha}=\int_{0}^{\pi} e^{i n \theta} \sigma_{N}^{\alpha}(\theta)^{2 k-1} \sin ^{m}(\theta / 2) d \theta
$$

where $k$ is any positive integer. If $0 \leq \alpha<(2 k-2) /(2 k-1)$ and $(2 k-1)(\alpha+1)-m \geq$ $\alpha$, we have

$$
I_{N}^{\alpha}=O\left(N^{2 k-2-m} \log N\right), \quad(N \rightarrow \infty) .
$$

If $0 \leq \alpha<(2 k-2) /(2 k-1)$ and $(2 k-1)(\alpha+1)-m<a$, we have

$$
I_{N}^{\alpha}=O\left(N^{-(2 k-1) \alpha-1+\alpha}\right) .
$$

If $-1<a<0$ and $(2 k-1)(\alpha+1)-m \geq 0$, we have

$$
I_{N}^{\alpha}=O\left(N^{2 k-2-m}\right), \quad(N \rightarrow \infty) .
$$

If $-1<\alpha<0$ and $(2 k-1)(\alpha+1)-m<0$, we have

$$
I_{N}^{\alpha}=O\left(N^{-\alpha(2 k-1)-1}\right), \quad(N \rightarrow \infty) .
$$


ProOF. Let

$$
I_{N}^{\alpha}=\left\{\int_{0}^{1 / N}+\int_{1 / N}^{\pi}\right\} e^{i \eta \theta}\left\{\sigma_{N}^{\alpha}(\theta)\right\}^{2 k-1} \sin ^{m}(\theta / 2) d \theta=\Delta_{1}+\Delta_{2} .
$$

By Lemma 2.8 it is easy to see that

$$
\Delta_{1}=O\left(N^{2 k-1} \int_{0}^{1 / N} \theta^{m} d \theta\right)=O\left(N^{2 k-2-m}\right)
$$

Applying (2.9) to $\Delta_{2}$, we obtain that the term $\Delta_{2}$ above is bounded by

$$
\begin{gathered}
O\left(N^{-\alpha(2 k-1)} \int_{1 / N}^{\pi} e^{i \eta \theta} \sin ^{2 k-1}\{(N+(\alpha+1) / 2) \theta-\alpha \pi / 2\} / \sin ^{(2 k-1)(\alpha+1)-m}(\theta / 2) d \theta\right) \\
+O\left(N^{-\alpha(2 k-2)-1} \int_{1 / N}^{\pi} \theta^{-(2 k-1)(\alpha+1)+\alpha-1+m} d \theta\right)=\Delta_{2.1}+\Delta_{2.2} .
\end{gathered}
$$

To estimate $\Delta_{2,1}$, letting $\left.(N+(\alpha+1) / 2)\right)=M$ and $-\alpha \pi / 2=\beta$, we can write

$$
\begin{aligned}
\sin ^{2 k-1}\{(N+(\alpha+1) / 2) \theta-\alpha \pi / 2\} & =C\left\{e^{-i(M \theta-\beta)}-e^{-i(M \theta-\beta)}\right\}^{2 k-1} \\
& =\sum_{l=0}^{2 k-1} C_{l} e^{i l(M \theta-\beta)} e^{-i(2 k-1-l)(M \theta-\beta)} \\
& =\sum_{l=0}^{2 k-1} B_{l} e^{i(2 l-2 k+1) M \theta},
\end{aligned}
$$

where the $C_{l}$ 's and $B_{l}$ 's are constants independent of $N$ and $\theta$. Without loss of generality we assume $M=N$ and $(2 l-2 k+1)=1$. Thus

$$
\Delta_{2,1}=O\left(N^{-\alpha(2 k-1)} \int_{1 / N}^{\pi} e^{i \eta \theta} e^{i N \theta} / \theta^{(2 k-1)(\alpha+1)-m} d \theta\right) .
$$

When $(2 k-1)(\alpha+1)-m \geq \alpha \geq 0$, using integration by parts we easily see that

$$
\Delta_{2,1}=O\left(N^{-\alpha(2 k-1)} N^{(2 k-1)(\alpha+1)-m-1}\right)=O\left(N^{2 k-2-m}\right) .
$$

Also since $(2 k-1)(\alpha+1)-m \geq \alpha \geq 0$ implies $-(2 k-1)(\alpha+1)+\alpha-1+m \leq-1$, we have

$$
\Delta_{2,2}=O\left(N^{2 k-2-m} \log N\right), \quad(N \rightarrow \infty) .
$$

Therefore (2.13) is proved.

To prove $\left(2.13^{\prime}\right)$, we again use (2.15). Noting that $m>(2 k-1)(\alpha+1)-\alpha$, by (2.16), we obtain $\Delta_{1}=O\left(N^{2 k-2-m}\right)=O\left(N^{2 k-2-(2 k-1)(\alpha+1)+\alpha}\right)=O\left(N^{-(2 k-1) \alpha-1+\alpha}\right)$. 
We write $\Delta_{2}=\Delta_{2.1}+\Delta_{2.2}$ as in (2.17). Since $(2 k-1)(\alpha+1)-m<\alpha$ implies $-(2 k-1)(\alpha+1)+\alpha+m-1>-1$, we easily see that $\Delta_{2,2}=O\left(N^{-\alpha(2 k-2)-1}\right)=$ $O\left(N^{-\alpha(2 k-1)-1+\alpha}\right)$. Using integration by parts and (2.18), we find that

$$
\Delta_{2.1}=O\left(N^{-\alpha(2 k-1)-1+(2 k-1)(\alpha+1)-m}\right)=O\left(N^{-\alpha(2 k-1)-1+\alpha}\right)
$$

since $m>(2 k-1)(\alpha+1)-\alpha$. Therefore $\left(2.13^{\prime}\right)$ is proved. If $-1<\alpha<0$ and $(2 k-1)(\alpha+1)-m \geq 0$, then $-(2 k-1)(\alpha+1)+m+\alpha-1 \leq \alpha-1<-1$. Thus by mimicking the proof of (2.13), one has no difficulty seeing $I_{N}^{\alpha}=O\left(N^{2 k-2-m}\right)$. This shows (2.14). If $-1<\alpha<0$ and $(2 k-1)(\alpha+1)-m<0$, we write $I_{N}^{\alpha}=\Delta_{1}+\Delta_{2}$ as in (2.15). By Lemma 2.8 , clearly

$$
\Delta_{1}=O\left(N^{2 k-2-m}\right)=O\left(N^{(2 k-2)-(2 k-1)(\alpha+1)}\right)=O\left(N^{-(2 k-1) \alpha-1}\right) .
$$

To estimate $\Delta_{2}$ we write $\Delta_{2}=\Delta_{2,1}+\Delta_{2.2}$ as in (2.17). Notice that $-(2 k-1)(\alpha+$ 1) $-(1-\alpha)+m<-(1-\alpha)$ and $1-\alpha>1$. We have

$$
\Delta_{2,2}=O\left(N^{-\alpha(2 k-2)-1} \int_{1 / N}^{\pi} \theta^{-(1-\alpha)} d \theta\right)=O\left(N^{-\alpha(2 k-1)-1}\right) .
$$

But using integration by parts, we also easily see that

$$
\Delta_{2,1}=O\left(N^{-\alpha(2 k-1)-1}\right) .
$$

Now (2.14) easily follows by (2.20), (2.21) and (2.22). The proof of Lemma 2.11 is complete.

\section{Proof of Theorem 1}

Let $\operatorname{Exp} \theta$ be an element in the maximal torus conjugate to $V \in \operatorname{SO}(2 k+1)$. From [G, p. 153] we know that $d(V, I)^{2}=\sum_{j=1}^{k} \sin \theta_{j} / 2$. From (1.5) we know that $K_{N}^{\alpha}(V)$ is a positive kernel. Thus by Lemma (2.2), it suffices to prove

$$
\lim _{N \rightarrow \infty} \int_{\mathrm{SO}(2 k+1)} \sum_{j=1}^{k} \theta_{j}^{2} K_{N}^{\alpha_{0}}(V) d V=0 .
$$

Noting that $\sum_{j=1}^{k} \theta_{j}^{2} K_{N}^{\alpha}(V)$ is a central function, from formula (1.5) and a symmetry argument we easily see that

$$
\int_{\mathrm{SO}(2 k+1)} \sum_{j=1}^{k} \theta_{j}^{2} K_{N}^{\alpha}(V) d V=\tilde{J}_{N}^{\alpha_{0}} / J_{N}^{\alpha_{0}},
$$


where

$$
\begin{aligned}
& J_{N}^{\alpha_{0}}=\int_{-\pi}^{\pi} \cdots \int_{-\pi}^{\pi} \prod_{j=1}^{k}\left|\sigma_{N}^{\alpha}\left(\theta_{j}\right)\right|^{2 k} D(\theta) d \theta_{1} \cdots d \theta_{k}, \\
& \tilde{J}_{N}^{\alpha_{0}}=\int_{-\pi}^{\pi} \cdots \int_{-\pi}^{\pi} \sum_{j=1}^{k} \theta_{j}^{2} \prod_{j=1}^{k}\left|\sigma_{N}^{\alpha}\left(\theta_{j}\right)\right|^{2 k} D(\theta) d \theta_{1} \cdots d \theta_{k},
\end{aligned}
$$

and

$$
D(\theta)=\prod_{j=1}^{k}\left(1-\cos \theta_{j}\right) \prod_{1 \leq i<j \leq k}\left(\cos \theta_{j}-\cos \theta_{i}\right)^{2}
$$

To prove (3.1), by Lemma 2.10 , it is enough to prove

$$
\tilde{J}_{N}^{\alpha_{0}}=O\left(N^{-k}\right)
$$

By using symmetry and changing variables, we only need to prove that

$$
\int_{0}^{\pi} \cdots \int_{0}^{\pi} \theta_{1}^{2} \prod_{j=1}^{k}\left|\sigma_{N}^{\alpha_{0}}\left(\theta_{j}\right)\right|^{2 k} D(\theta) d \theta_{1} \cdots d \theta_{k}=O\left(N^{-k}\right)
$$

By symmetry again, we need to prove

$$
\begin{aligned}
\int_{0}^{1 / N} & \cdots \int_{0}^{1 / N} \theta_{1}^{2} \prod_{j=1}^{k}\left|\sigma_{N}^{\alpha_{0}}\left(\theta_{j}\right)\right|^{2 k} D(\theta) d \theta \\
& +\int_{1 / N}^{\pi} \cdots \int_{1 / N}^{\pi} \theta_{1}^{2} \prod_{j=1}^{k}\left|\sigma_{N}^{\alpha_{0}}\left(\theta_{j}\right)\right|^{2 k} D(\theta) d \theta \\
& +\sum_{\mu=1}^{k-1}\left\{\int_{1 / N}^{\pi} \ldots \int_{1 / N}^{\pi}\right\}\left\{\int_{0}^{1 / N} \cdots \int_{(\mu)}^{1 / N}\right\} \theta_{1}^{2} \prod_{j=1}^{k}\left|\sigma_{N}^{\alpha_{0}}\left(\theta_{j}\right)\right|^{2 k} D(\theta) d \theta \\
& \left.+\sum_{\mu=1}^{k-1}\left\{\int_{0}^{1 / N} \cdots \int_{0}^{1 / N}\right\}\left\{\int_{1 / N}^{\pi} \int_{1 / N}^{\pi}\right\}\right) \theta_{1}^{2} \prod_{j=1}^{k}\left|\sigma_{N}^{\alpha_{0}}\left(\theta_{j}\right)\right|^{2 k} D(\theta) d \theta_{1} \cdots d \theta_{k} \\
= & J_{1}+J_{2}+\sum_{\mu=1}^{k-1} J^{(\mu)}+\sum_{\mu=1}^{k-1} J_{(\mu)}=O\left(N^{-k}\right) .
\end{aligned}
$$

First by Lemma 2.8 we easily see that $J_{1}=O\left(N^{-k-2}\right)=O\left(N^{-k}\right)$. To estimate the 
remaining terms, we notice that

$$
\begin{aligned}
D(\theta) & =\prod_{j=1}^{k}\left(1-\cos \theta_{j}\right) \sum_{1 \leq i<j \leq k}\left\{\left(1-\cos \theta_{i}\right)-\left(1-\cos \theta_{j}\right)\right\}^{2} \\
& =O\left(\prod_{j=1}^{k} \theta_{j}^{2} \sum_{1 \leq i<j \leq k}\left\{\theta_{i}^{2}+\theta_{j}^{2}\right\}\right)=O\left(\sum_{(p)} \prod_{j=1}^{k} \theta_{j}^{p_{j}}\right),
\end{aligned}
$$

where $(p)=\left(p_{1}, \ldots, p_{k}\right)$ is a $k$-tuple of even integers between 2 and $4 k-2$, $\sum_{j=1}^{k} p_{j}=2 k^{2}$, and for each $(p)$, at most one $p_{j}$ is equal to $4 k-2$. Also by Lemma 2.8, $\prod_{j=1}^{k}\left|\sigma_{N}^{\alpha_{0}}\left(\theta_{j}\right)\right|^{2 k}=O\left(N^{k(1-2 k)} \prod_{j=1}^{k} \theta_{j}^{-4 k+1}\right)$. Thus we find that $J_{2}$ is equal to

$$
O\left(\sum_{(p)} N^{k(1-2 k)} \int_{1 / N}^{\pi} \cdots \int_{1 / N}^{\pi} \theta_{1}^{-4 k+3+p_{1}} \prod_{j=2}^{k} \theta_{j}^{-4 k+1+p_{j}} d \theta_{1} \cdots d \theta_{k}\right) .
$$

If $p_{1}$ is $4 k-2$, then we easily see that $\sum_{j=2}^{k} p_{j}=2(k-1)^{2}$ and $p_{j} \leq 4 k-4$ for all $j=2,3, \ldots, k$. After this observation, using Lemma 2.8 , we easily calculate that for this $(p)$ the term in (3.9) is equal to

$$
\begin{aligned}
N^{k(1-2 k)} & \int_{1 / N}^{\pi} \cdots \int_{1 / N}^{\pi} \theta_{1} \prod_{j=2}^{k} \theta_{j}^{-4 k+1+p_{j}} d \theta_{1} \cdots d \theta_{k} \\
= & O\left(N^{k(1-2 k)-2(k-1)^{2}+(k-1)(4 k-2)}\right)=O\left(N^{-k}\right) .
\end{aligned}
$$

In a similar way, we estimate each term in (3.9) by considering the different cases $p_{1}=4 k-4$, and $p_{1}<4 k-4$. It is easy to see that each term in (3.9) is $O\left(N^{-k}\right)$ as $N \rightarrow \infty$. This shows $J_{2}=O\left(N^{-k}\right)$. Next we estimate each term $J^{(\mu)}$ in (3.8). We write

$$
D\left(\theta_{1}, \theta_{2}, \ldots, \theta_{k}\right)=D\left(\theta_{\mu+1}, \ldots, \theta_{k}\right)\left\{D\left(\theta_{1}, \ldots, \theta_{k}\right) / D\left(\theta_{\mu+1}, \ldots, \theta_{k}\right)\right\} .
$$

By Lemma 2.8,

$$
\begin{aligned}
& \boldsymbol{J}^{(\mu)}= o\left(N^{2 k(k-\mu)} \int_{0}^{1 / N} \cdots \int_{0}^{1 / N} D\left(\theta_{\mu+1}, \ldots, \theta_{k}\right)\right. \\
&\left\{\int_{1 / N}^{\pi} \cdots \int_{1 / N}^{\pi} \theta_{1}^{2} \prod_{j=1}^{\mu}\left|\sigma_{N}^{\alpha_{0}}\left(\theta_{j}\right)\right|^{2 k} D\left(\theta_{1}, \ldots, \theta_{k}\right) / D\left(\theta_{\mu+1}, \ldots, \theta_{k}\right) d \theta_{1} \cdots d \theta_{\mu}\right\} \\
&\left.d \theta_{\mu+1} \cdots d \theta_{k}\right)
\end{aligned}
$$


where

$$
\int_{0}^{1 / N} \cdots \int_{0}^{1 / N} D\left(\theta_{\mu+1}, \ldots, \theta_{k}\right) d \theta_{\mu+1} \cdots d \theta_{k}=O\left(N^{-(k-\mu)(2 k-2 \mu+1)}\right)
$$

since $(k-\mu)(2 k-2 \mu+1)$ is the dimension of $\operatorname{SO}(2(k-\mu)+1)$.

Notice that as a polynomial in the terms $\left(1-\cos \theta_{j}\right), D\left(\theta_{1}, \ldots, \theta_{k}\right) / D\left(\theta_{\mu+1}, \ldots, \theta_{k}\right)$ is a homogeneous polynomial with

$$
\begin{gathered}
\text { degree }\left\{D\left(\theta_{1}, \ldots, \theta_{k}\right) / D\left(\theta_{\mu+1}, \ldots, \theta_{k}\right)\right\}=\mu(2 k-\mu) \\
=\text { degree } D\left(\theta_{1}, \ldots, \theta_{k}\right)-\text { degree } D\left(\theta_{\mu+1}, \ldots, \theta_{k}\right) .
\end{gathered}
$$

Thus by definition,

$$
D\left(\theta_{1}, \ldots, \theta_{k}\right) / D\left(\theta_{\mu+1}, \ldots, \theta_{k}\right)=\sum_{(l)} O\left(\prod_{j=1}^{\mu}\left(1-\cos \theta_{j}\right)^{l_{j}}\right)
$$

+ terms of homogeneous degree $\mu(2 k-\mu)$ that contain at least one $\left(1-\cos \theta_{j}\right)$ with $j \in\{\mu+1, \ldots, k\}$, where $(l)=\left(l_{1}, \ldots, l_{\mu}\right)$ is a $\mu$-tuple such that $\sum l_{j}=\mu(2 k-\mu)$.

For $\left(\theta_{1}, \ldots, \theta_{\mu}\right) \in(1 / N, \pi]^{\mu}$ and $\left(\theta_{\mu+1}, \ldots, \theta_{k}\right) \in[0,1 / N)^{k-\mu}$, since $\left(1-\cos \theta_{j}\right)=O\left(N^{-2}\right)$ if $j \in\{\mu+1, \ldots, k\}$, without loss of generality we can assume

$$
D\left(\theta_{1}, \ldots, \theta_{k}\right) / D\left(\theta_{\mu+1}, \ldots, \theta_{k}\right)=O\left(\sum_{(l)} \prod_{j=1}^{\mu} \theta_{j}^{2 l_{j}}\right) .
$$

By Lemma 2.8,

$$
\prod_{j=1}^{\mu}\left|\sigma_{N}^{\alpha_{0}}\left(\theta_{j}\right)\right|^{2 k}=O\left(N^{-\mu(2 k-1)} \prod_{j=1}^{\mu} \theta_{j}^{1-4 k}\right) .
$$

Thus a simple computation shows that

$$
\begin{aligned}
J^{(\mu)} & =O\left(\sum_{(P)} N^{-k+2 \mu(1-\mu)} \int_{1 / N}^{\pi} \theta_{1}^{-4 k+3+p_{1}} \prod_{j=2}^{\mu} \int_{1 / N}^{\pi} \theta_{j}^{-4 k+1-p_{j}} d \theta_{j}\right) \\
& =o\left(\sum_{(p)} J^{(\mu)}(p)\right) .
\end{aligned}
$$

In the above equality, $\sum_{(p)}$ is a sum with finite number of terms, namely $\sum_{j=1}^{\mu} p_{j}=$ $2 \mu(2 k-\mu)$; these $p_{j}$ 's have the same properties as we discussed in (3.9). Now we estimate these terms $J^{(\mu)}(p)$ in the following different cases that include all the possible cases of $J^{(\mu)}(p)$.

(1) $p_{1}=4 k-2$, all other $p_{j} \leq 4 k-4(j=2, \ldots, \mu)$. 
(2) $p_{1}=4 k-4$, there is one $p_{j}=4 k-2$.

(3) $p_{1}=4 k-4$, all other $p_{j}$ 's are less than or equal to $4 k-4$.

(4) $p_{1}=4 k-6$, there is one $p_{j}=4 k-2$.

(5) $p_{1}=4 k-6$, all other $p_{j}$ 's are less than or equal to $4 k-4$.

(6) $p_{1}<4 k-6$, all other $p_{j}$ 's are less than or equal to $4 k-4$.

(7) $p_{1}<4 k-6$, there is one $p_{j}=4 k-2$.

In the case (1), noting that $\sum_{j=2}^{\mu} p_{j}=2 \mu(2 k-\mu)-4 k+2$, we easily compute that

$$
\begin{aligned}
J^{(\mu)}(p) & =O\left(N^{-k+2 \mu(1-\mu)} \prod_{j=2}^{\mu} \int_{1 / N}^{\pi} \theta_{j}^{-4 k+1+p_{j}} d \theta_{j}\right) \\
& =O\left(N^{-k+2 \mu(1-\mu)} N^{(4 k-2)(\mu-1)-2 \mu(2 k-\mu)+4 k-2}\right)=O\left(N^{-k}\right) .
\end{aligned}
$$

In a similar manner, it is easy to compute that $J^{(\mu)}(p)=O\left(N^{-k}\right)$ in the other different cases. Finally we estimate each $J_{(\mu)}$ in (3.8). It is easy to see

$$
\begin{aligned}
J_{(\mu)} & =\left\{\int_{0}^{1 / N} \cdots \int_{0}^{1 / N}\right\}\left\{\int_{1 / N}^{\pi} \cdots \int_{1 / N}^{\pi}\right\} \theta_{1}^{2} \prod_{j=1}^{k}\left|\sigma_{N}^{\alpha_{0}}\left(\theta_{j}\right)\right|^{2 k} D(\theta) d \theta \\
& \leq\left\{\int_{0}^{1 / N} \cdots \int_{0}^{1 / N}\right\}\left\{\int_{1 / N}^{\pi} \cdots \int_{1 / N}^{\pi}\right\} \theta_{k}^{2} \prod_{j=1}^{k}\left|\sigma_{N}^{\alpha_{0}}\left(\theta_{j}\right)\right|^{2 k} D(\theta) d \theta .
\end{aligned}
$$

But $\prod_{j=1}^{k}\left|\sigma_{N}^{\alpha_{0}}\left(\theta_{j}\right)\right|^{2 k} D(\theta)$ is a symmetric function. So it is reduced to estimating the previous case $J^{(\mu)}$. Theorem 1 is proved.

\section{Proofs of Theorem 3 and Theorem 4}

Remember that $\Delta(\theta)=\prod_{1 \leq i<j \leq k}\left(\cos \theta_{i}-\cos \theta_{j}\right)^{2}$. By the definition of the Cesàro kernel on rotation group $\mathrm{SO}(2 k)$, from (1.4) and a symmetry argument we know that

$$
\int_{\mathrm{SO}(2 k)}\left|K_{N}^{\alpha}(V)\right| d V=\mathscr{J}^{\alpha} / \mathscr{I}^{\alpha},
$$

where

$$
\mathscr{J}^{\alpha}=\int_{-\pi}^{\pi} \cdots \int_{-\pi}^{\pi} \prod_{j=1}^{k}\left|\sigma_{N}^{\alpha}\left(\theta_{j}\right)\right|^{2 k-1} \Delta(\theta) d \theta_{1} \cdots d \theta_{k}
$$

and

$$
\mathscr{I}^{\alpha}=\int_{-\pi}^{\pi} \cdots \int_{-\pi}^{\pi} \prod_{j=1}^{k}\left\{\sigma_{N}^{\alpha}\left(\theta_{j}\right)\right\}^{2 k-1} \Delta(\theta) d \theta_{1} \cdots d \theta_{k}
$$


By Lemma $2.10^{\prime}$, we know that $\mathscr{J}^{\alpha_{0}} \geq A \log N$ for sufficiently large $\mathrm{N}$. So to prove Theorem 3 , it suffices to prove $\mathscr{I}^{\alpha_{0}}=O(1)$. By the definition of $\Delta(\theta)$, after changing variables it suffices to prove that

$$
\sum_{(p)} \prod_{j=1}^{k} \int_{0}^{\pi}\left\{\sigma_{N}^{\alpha_{0}}\left(\theta_{j}\right)\right\}^{2 k-1} \theta_{j}^{p_{j}} d \theta_{j}=O(1)
$$

where the $p_{j}$ 's are even integers between 0 and $4 k-4$ and there is at most one $p_{j}$ which is $4 k-4, \sum_{(p)}$ is a summation of a finite number of terms with $\sum_{j=1}^{k} p_{j}=2 k(k-1)$. By symmetry, we need only prove that

$$
\begin{aligned}
& E_{1}=\prod_{j=1}^{k} \int_{0}^{1 / N}\left\{\sigma_{N}^{\alpha_{0}}\left(\theta_{j}\right)\right\}^{2 k-1} \theta_{j}^{p_{j}} d \theta_{j}=O(1), \\
& E_{2}=\prod_{j=1}^{k} \int_{1 / N}^{\pi}\left\{\sigma_{N}^{\alpha_{0}}\left(\theta_{j}\right)\right\}^{2 k-1} \theta_{j}^{p_{j}} d \theta_{j}=O(1), \\
& E_{3}=\prod_{j=1}^{k} \int_{0}^{1 / N}\left\{\sigma_{N}^{\alpha_{0}}\left(\theta_{j}\right)\right\}^{2 k-1} \theta_{j}^{p_{j}} d \theta_{j} \prod_{j=\mu+1}^{k} \int_{1 / N}^{\pi} \sigma_{N}^{\alpha_{0}}\left(\theta_{j}\right)^{2 k-1} \theta_{j}^{p_{j}} d \theta_{j}=O(1),
\end{aligned}
$$

$(\mu=1,2, \ldots, k-1)$ for all $(p)=\left(p_{1}, \ldots, p_{k}\right)$ satisfying the conditions in (4.2). By Lemma 2.8 together with the conditions of $(p)$, one easily sees that $E_{1}=O\left(N^{-2 k(k-1)-k+k(2 k-1)}\right)=O(1)$. Now we estimate $E_{2}$. If all $p_{j}$ 's are less than $4 k-4$, then $E_{2}$ is equal to

$$
O\left(\prod_{j=1}^{k} N^{-k(2 k-2)} \int_{1 / N}^{\pi} \theta_{j}^{-4 k+3+p_{j}} d \theta_{j}\right)=O\left(N^{-k(2 k-2)+k(4 k-4)-2 k(k-1)}\right)=O(1) .
$$

If there is one $p_{j}$ which equals $4 k-4$, without loss of generality we assume $p_{1}=4 k-4$; then $\sum_{j=2}^{k} p_{j}=2 k(k-1)-4 k+4=2 k^{2}-6 k+4$. By (2.9) we obtain that $E_{2}$ is equal to

$$
O\left(N^{-2(k-2)(k-1)} \int_{1 / N}^{\pi} \sigma_{N}^{\alpha_{0}}\left(\theta_{1}\right)^{2 k-1} \theta_{1}^{4 k-4} d \theta_{1} N^{(k-1)(4 k-4)-\left(2 k^{2}-6 k+4\right)}\right) .
$$

Again by (2.9) together with a simple computation, we know that the above equality is equal to

$$
\begin{gathered}
O\left(N^{2 k-2} N^{-2 k+2} \int_{1 / N}^{\pi} \sin ^{2 k-1}\left\{\left(N+1 / 2+\alpha_{0}\right) \theta_{1}-\alpha_{0} \pi / 2\right\} / \theta_{1} d \theta_{1}\right) \\
+O\left(N^{-1 /(2 k-1)} \int_{1 / N}^{\pi} \theta^{-1-1 /(2 k-1)} d \theta\right)=O(1)
\end{gathered}
$$


To estimate $E_{3}$, if all $p_{j}$ 's are less than $4 k-4$ or if one of $p_{1}, p_{2}, \ldots, p_{\mu}$ is $4 k-4$, one easily obtains, following the same estimates as in $E_{1}$ and $E_{2}$, that

$$
E_{3}=O\left(N^{2 \mu(k-1)} N^{4(k-\mu)(k-1)} N^{-2(k-\mu)(k-1)} N^{-2 k(k-1)}\right)=O(1) .
$$

If one of $p_{\mu+1}, \ldots, p_{k}$ is $4 k-4$, we may assume $p_{k}=4 k-4$. After a simple computation we know that $E_{3}$ is equal to

$$
\begin{aligned}
& O\left(N^{\mu(2 k-1)-\mu-(2 k-2)(k-1-\mu)+(4 k-4)(k-\mu-1)-\sum_{j=1}^{k-1} p_{j}}\right) \int_{1 / N}^{\pi} \sigma_{N}^{\alpha_{0}}(\theta)^{2 k-1} \theta^{4 k-4} d \theta \\
& =O\left(N^{(2 k-1)} \int_{1 / N}^{\pi}\left\{\sigma_{N}^{\alpha_{0}}(\theta)\right\}^{2 k-1} \theta^{4 k-4} d \theta\right) .
\end{aligned}
$$

Thus the estimates (4.4) and (4.5) show that $E_{3}=O(1)$. This completes the proof of Theorem 3.

Now we are going to prove Theorem 4 . Noting that the Lebesgue constant in this case is formulated by (4.1), we need to estimate both $\mathscr{J}^{\alpha}$ and $\mathscr{I}^{\alpha}$ for $-1<\alpha<$ $(2 k-2) /(2 k-1)$. We will consider the case $0 \leq \alpha<(2 k-2) /(2 k-1)$ only, since the proof for the case $-1<\alpha<0$ is similar. It is easy to see that $\mathscr{J}^{\alpha}$ is bounded below by

$$
\begin{gathered}
\int_{\pi / 4 \geq \theta_{1} \geq 2 \theta_{2} \geq \cdots \geq 2^{k-1} \theta_{k} \geq 0}\left|\prod_{j=1}^{k} \sigma_{N}^{\alpha}\left(\theta_{j}\right)\right|^{2 k-1} \prod_{1 \leq i<j \leq k} \sin ^{2}\left(\left(\theta_{i}-\theta_{j}\right) / 2\right) \sin ^{2}\left(\left(\theta_{i}+\theta_{j}\right) / 2\right) d \theta \\
\geq A \int_{\pi / 4 \geq \theta_{1} \geq \cdots \geq 2^{k-1} \theta_{k} \geq 0}\left|\prod_{j=1}^{k} \sigma_{N}^{\alpha}\left(\theta_{j}\right)\right|^{2 k-1} \theta_{1}^{4(k-1)} \theta_{2}^{4(k-2)} \theta_{k-1}^{4} d \theta_{1} \cdots d \theta_{k} .
\end{gathered}
$$

The last inequality is true since for any $i<j$,

$$
\sin ^{2}\left(\left(\theta_{i}-\theta_{j}\right) / 2\right) \sin ^{2}\left(\left(\theta_{i}+\theta_{j}\right) / 2\right) \geq A\left(\theta_{i}^{2}-\theta_{j}^{2}\right) \geq(A / 2) \theta_{i}^{2} .
$$

Now we let $\xi=k-[((2 k-1)(\alpha+1)+3) / 4]=k-[\gamma]$, where $[\gamma]$ means the integer part of $\gamma=((2 k-1)(\alpha+1)+3) / 4$. Because $-1<\alpha<(2 k-2) /(2 k-1)$ and $\gamma-1<[\gamma] \leq \gamma$, one easily sees that

$$
\begin{array}{ll}
0 \leq \xi \leq k, \quad(2 k-1)(\alpha+1)-4(k-\xi)<1 \quad \text { and } \\
& (2 k-1)(\alpha+1)-4(k-\xi-1) \geq 1 .
\end{array}
$$

In fact, since $\gamma=\{(2 k-1)(\alpha+1)+3\} / 4$, we have $(2 k-1)(\alpha+1)-4(k-\xi)=$ $(2 k-1)(\alpha+1)-4[\gamma]<(2 k-1)(\alpha+1)-4 \gamma+4=1$ and $(2 k-1)(\alpha+1)-4(k-\xi-1)=$ 
$(2 k-1)(\alpha+1)-4([\gamma]-1)>(2 k-1)(\alpha+1)-4 \gamma+4=1$. This shows (4.6). Let $\Omega_{1}$ denote the expression

$$
\int_{1 / N \geq 2^{\xi} \theta_{\xi+1} \geq \cdots \geq 2^{k-1} \theta_{k} \geq 0}\left|\prod_{j=\xi+1}^{k} \sigma_{N}^{\alpha}\left(\theta_{j}\right)\right|^{2 k-1} \theta_{\xi+1}^{4(k-\xi-1)} \cdots \theta_{k-1}^{4} d \theta_{\xi+1} \cdots d \theta_{k}
$$

for $1 \leq \xi \leq k-1$ and $\Omega_{1}=1$ if $\xi=k$

$$
\Omega_{2}=\int_{\pi / 4 \geq \theta_{1} \geq \cdots \geq 2^{\xi-1} \theta_{\xi} \geq 1 / N} \prod_{j=1}^{\xi}\left\{\left|\sigma_{N}^{\alpha}\left(\theta_{j}\right)\right|^{2 k-1} \theta_{j}^{4(k-j)}\right\} d \theta_{1} \cdots d \theta_{\xi}
$$

for $\xi \neq 0$, and $\Omega_{2}=1$ if $\xi=0$. Clearly $\mathscr{J}^{\alpha} \geq \Omega_{1} \Omega_{2}$.

By Lemma 2.8, it is obvious that

$$
\Omega_{1} \geq A N^{(2 k-1)(k-\xi)-(k-\xi)-4(1+2+\cdots+(k-\xi-1)\}}=A N^{2 \xi(k-\xi)} .
$$

By using formula (2.9), a simple computation shows that $\Omega_{2}$ is greater than or equal to

$$
\begin{aligned}
& A N^{-(2 k-1) \alpha \xi} \int_{\pi / 4 \geq \theta_{1} \geq \cdots \geq 2^{\xi-1} \theta_{\xi} \geq 1 / N} \prod_{j=1}^{\xi} \frac{\left|\sin ^{2 k-1}\left\{(N+1 / 2+\alpha / 2) \theta_{j}-\alpha \pi / 2\right\}\right|}{\left\{\sin \left(\theta_{j} / 2\right)\right\}^{(2 k-1)(\alpha+1)-4(k-j)}} d \theta_{1} \cdots d \theta_{\xi} \\
& \quad+o\left(N^{-(2 k-1) \alpha \xi}\right) .
\end{aligned}
$$

By the choice of this $\xi$, for $j=1,2, \ldots, \xi$ we have $(2 k-1)(\alpha+1)-4(k-j) \leq$ $(2 k-1)(\alpha+1)-4(k-\xi)<1$. Thus clearly we see that $\Omega_{2} \geq A N^{-(2 k-1) \alpha \xi}$, as $N \rightarrow \infty$. We now obtain that

$$
\mathscr{J}^{\alpha} \geq A \Omega_{1} \Omega_{2} \geq A N^{2 \xi(k-\xi)-(2 k-1) \alpha \xi} .
$$

Next we estimate $\mathscr{I}^{\alpha}$. By the definitions of $\mathscr{I}^{\alpha}$ and $\Delta(\theta)$, after changing variables, we obtain that the absolute value of $\mathscr{I}^{\alpha}$ is bounded by

$$
A \sum_{(m)} \sum_{(q)}\left|\prod_{j=1}^{k} \int_{0}^{\pi}\left\{\sigma_{N}^{\alpha_{0}}\left(\theta_{j}\right)\right\}^{2 k-1} \sin ^{m_{j}+q_{j}}\left(\theta_{j} / 2\right) d \theta_{j}\right|=\sum_{(m)} \sum_{(q)} \Delta_{m, q} .
$$

In formula (4.8), $\sum_{(m)}$ and $\sum_{(q)}$ are summations with a finite number of terms; both $(m)=\left(m_{1}, m_{2}, \ldots, m_{k}\right)$ and $(q)=\left(q_{1}, q_{2}, \ldots, q_{k}\right)$ are permutations of $(0,2, \ldots$, $2(k-1))$. Thus without loss of generality, we may assume $0 \leq m_{1}+q_{1} \leq m_{2}+q_{2} \leq$ $\cdots \leq m_{k}+q_{k} \leq 4(k-1)$. We estimate these $\Delta_{m, q}$ 's in (4.8) in the following different cases. 
CASE 1. If $(2 k-1)(\alpha+1)-m_{k}-q_{k} \geq \alpha$, then $(2 k-1)(\alpha+1)-m_{j}-q_{j} \geq \alpha$ for all $j=1,2, \ldots, k$. In this case we use formula (2.13) to obtain

$$
\Delta_{m, q} \leq A N^{(2 k-2) k-\sum_{j=1}^{k}\left(m_{j}+q_{j}\right)} \log ^{k} N .
$$

But we know $\sum_{j=1}^{k} m_{j}+q_{j}=k(2 k-2)$. This implies that

$$
\Delta_{m, q} \leq A \log ^{k} N \quad(N \rightarrow \infty) .
$$

CASE 2. If $(2 k-1)(\alpha+1)-m_{j}-q_{j}<\alpha$ for all $j=1, \ldots, k$, then by $\left(2.13^{\prime}\right)$ we have

$$
\Delta_{m, q} \leq A N^{-2 k^{2} \alpha+2 \alpha k-k} .
$$

CASE 3. There exists an integer $j_{0}$, which is bounded below by 1 and above by $k-1$, such that

$$
\begin{gathered}
(2 k-1)(\alpha+1)-\left(m_{j_{0}}+q_{j_{0}}\right) \geq \alpha, \\
(2 k-1)(\alpha+1)-\left(m_{j_{0}+1}+q_{j_{0}+1}\right)<\alpha .
\end{gathered}
$$

By the choice of $(m)$ and $(q)$ we know that

$$
\sum_{j=1}^{j_{0}}\left(m_{j}+q_{j}\right) \geq 2 j_{0}\left(j_{0}-1\right) .
$$

By (2.13) we know that

$$
\Delta_{m, q} \leq A N^{j_{0}(2 k-2)-\sum_{j=1}^{j_{0}}\left(m_{j}+q_{j}\right)} \log ^{j_{0}} N \prod_{j=j_{0}+1}^{k} \omega_{j}(m, q),
$$

and

$$
\omega_{j}(m, q)=\int_{0}^{\pi}\left\{\sigma_{N}^{\alpha}\left(\theta_{j}\right)\right\}^{2 k-1} \sin ^{m_{j}+q_{j}}\left(\theta_{j} / 2\right) d \theta_{j} .
$$

So by (2.9) and $\left(2.13^{\prime}\right)$ we obtain that

$$
\omega_{j}(m, q)=O\left(N^{-\left(k-j_{0}\right)\{(2 k-1) \alpha-\alpha+1\}}\right),
$$

which leads to

$$
\Delta_{m, q} \leq A N^{\left(k-j_{0}\right)\left(2 j_{0}-2 k \alpha+2 \alpha-1\right)} \log ^{j_{0}} N
$$


Combining Cases 1 to 3 , we easily see that the above inequality (4.15) is true for all $j_{0}=0,1, \ldots, k$. Now we estimate the maximum value of $\left(k-j_{0}\right)\left(2 j_{0}-2 k \alpha+2 \alpha-1\right)$ for $j_{0}=0,1, \ldots, k$. Consider the quadratic function

$$
Q(x)=(k-x)(2 x-2 k \alpha+2 \alpha-1) \quad x \in[0, k] .
$$

It is easy to see that $Q(x)$ reaches its unique maximum value at the point $\beta=$ $(2 k \alpha-2 \alpha+1+2 k) / 4<k$. Notice that $Q(x)$ being a quadratic function implies that for all $j_{0}=0,1, \ldots, k$,

$$
\text { (4.17) } \begin{aligned}
\left(k-j_{0}\right)\left(2 j_{0}-2 k \alpha+2 \alpha-1\right) \leq \max \{(k-[\beta])(2[\beta]-2 k \alpha+2 \alpha-1) \\
(k-[\beta]-1)(2[\beta]-2 k \alpha+2 \alpha+1)\} .
\end{aligned}
$$

Thus by (4.8), (4.9') and (4.15), we have

$$
\mathscr{I}^{\alpha} \leq A \log ^{k} N \max \left\{N^{(k-[\beta])(2[\beta]-2 k \alpha+2 \alpha-1)}, N^{(k-[\beta]-1)(2[\beta]-2 k \alpha+2 \alpha+1)}\right\} .
$$

Now by the definition of the Lebesgue constant together with (4.7) we know that when $0 \leq \alpha<(2 k-2) /(2 k-1)$ the Lebesgue constant of the Cesàro mean on $\mathrm{SO}(2 k)$ is

$$
\mathscr{J}^{\alpha} / \mathscr{I}^{\alpha} \geq A \log ^{-k} N \min \left\{N^{F(k, \alpha)}, N^{G(k, \alpha)}\right\}
$$

where

$$
\begin{aligned}
& F(k, \alpha)=(k-[\gamma])(2[\gamma]-2 k \alpha+\alpha)-(k-[\beta])(2[\beta]-2 k \alpha+2 \alpha-1), \\
& G(k, \alpha)=(k-[\gamma])(2[\gamma]-2 k \alpha+\alpha)-(k-[\beta]-1)(2[\beta]-2 k \alpha+2 \alpha+1)
\end{aligned}
$$

and $\gamma=\{(2 k-1)(\alpha+1)+3\} / 4$. Since $\gamma=\beta+(\alpha+1) / 4$ and $|\alpha|<1$, we have either $[\gamma]=[\beta]$ or $[\gamma]=[\beta]+1$. Therefore to finish proving the theorem, it suffices to show that

$$
F(k, \alpha)>0 \text { and } G(k, \alpha)>0 .
$$

In fact, if $[\gamma]=[\beta]$, then because $\alpha<(2 k-2) /(2 k-1)$,

$$
\begin{aligned}
F(k, \alpha) & =(1-\alpha)(k-[\gamma]) \geq(1-\alpha)(k-\gamma) \\
& =(1-\alpha)\{(2 k-1)(1-\alpha)-1\} / 2>0 .
\end{aligned}
$$

If $[\gamma]-1=[\beta]$, then a simple computation shows that

$$
\begin{aligned}
F(k, \alpha) & =(k-[\gamma])(2[\gamma]-2 k \alpha+\alpha)-\{k-[\gamma]+1\}\{2[\gamma]-(2 k-1) \alpha+\alpha-3\} \\
& =(1-\alpha)(k-[\gamma])+2(k-[\gamma])-2[\gamma]+\alpha(2 k-1)-\alpha+3 .
\end{aligned}
$$


Since $(1-\alpha)(k-[\gamma])>0$ from the previous proof, we need only show that

$$
2(k-[\gamma])-2[\gamma]+\alpha(2 k-1)-\alpha+3>0 .
$$

In fact $2(k-[\gamma])-2[\gamma]+\alpha(2 k-1)-\alpha+3=2 k-4[\gamma]+\alpha(2 k-1)-\alpha+3 \geq$ $2 k-4 \gamma+\alpha(2 k-1)-\alpha+3=2 k-(2 k-1)(\alpha+1)+\alpha(2 k-1)-\alpha=(1-\alpha)>0$. This shows $\mathrm{F}(\mathrm{k}, \mathrm{a})>0$.

Next we show that $G(k, a)>0$. By the definition of $G(k, \alpha)$, if $[\beta]=[\gamma]$ then from the proof for $F(k, \alpha)$ we easily see that $G(k, \alpha)>0$. If $[\beta]=[\gamma]-1$ then a simple computation shows that $G(k, \alpha)=(k-[\gamma])(1-\alpha)-2 k+4[\gamma]-(2 k-1) \alpha+1+\alpha$. Again, to prove $G(k, \alpha)>0$ we only need to prove that

$$
-2 k+4[\gamma]-(2 k-1) \alpha+1+\alpha>0 .
$$

Remember $[\gamma]=[\beta]+1,[\beta] \geq \beta-1$ and $\gamma=\beta+(1+\alpha) / 4$. We now have

$$
\begin{aligned}
& -2 k+4[\gamma]-(2 k-1) \alpha+1+\alpha=-2 k+4[\beta]+4-(2 k-1) \alpha+1+\alpha \\
& \quad \geq-2 k+4 \gamma-(2 k-1) \alpha=-2 k+(2 k-1)(\alpha+1)-(2 k-1) \alpha+3>0 .
\end{aligned}
$$

This shows $G(k, \alpha)>0$. Thus (4.19) is proved. Now we can find an $\epsilon>0$, such that when $0 \leq a<(2 k-2) /(2 k-1)$,

$$
\int_{\text {SO(2k) }}\left|K_{N}^{\alpha}(V)\right| d V \geq A N^{\epsilon}, \quad N \rightarrow \infty .
$$

Using the same argument, replacing (2.13) and $\left(2.13^{\prime}\right)$ by $(2.14)$ and $\left(2.14^{\prime}\right)$, we can prove that formula (4.20) also holds for $-1<\alpha<0$. We leave these details to the reader.

NoTE. On SO(4), we obtained some estimates which are more precise than (1.9). The details can be found in $[F 1]$.

\section{Proof of Theorem 2}

Recall that any $V \in \operatorname{SO}(2 k+1)$ is conjugate to a $(2 k+1) \times(2 k+1)$ matrix $\operatorname{diag}\left(S\left(\theta_{1}\right), S\left(\theta_{2}\right), \ldots, S\left(\theta_{k}\right), 1\right)$. Clearly $g(V)=\left(1-\cos \theta_{1}\right)$ is a $C^{\infty}$ function and $g(I)=0$. Now we shall prove that the function $g(V)$ furnishes Theorem 2. Since the case $k=1$ is proved in [F1], we will only prove the case $k \geq 2$. By formula (1.5), we know that

$$
\int_{\mathrm{SO}(2 k+1)} K_{N}^{\alpha}(V) d V=\Delta^{\alpha} / I^{\alpha}
$$


where

$$
\Delta^{\alpha}=\int_{-\pi \leq \theta_{k} \leq \cdots \leq \theta_{1} \leq \pi} \ldots \int_{j=1}\left(1-\cos \theta_{1}\right) \prod_{j=1}^{k}\left\{\sigma_{N}^{\alpha}\left(\theta_{j}\right)\right\}^{2 k} D\left(\theta_{1}, \ldots, \theta_{k}\right) d \theta_{1} \cdots d \theta_{k}
$$

and

$$
I^{\alpha}=\int_{-\pi \leq \theta_{k} \leq \cdots \leq \theta_{1} \leq \pi} \ldots \int_{N}\left\{\sigma_{N}^{\alpha}\left(\theta_{j}\right)\right\}^{2 k} D\left(\theta_{1}, \ldots, \theta_{k}\right) d \theta_{1} \cdots d \theta_{k}
$$

For any fixed $\alpha \in(-1,(2 k-1) /(2 k))$, there exists a natural number $\zeta \in\{1,2, \ldots, k\}$ such that

$$
\begin{gathered}
\zeta_{\alpha}=2 k(\alpha+1)-2-4(k-\zeta) \leq 1, \\
2 k(\alpha+1)-2-4(k-\zeta-1)>1
\end{gathered}
$$

Thus using the same argument as we did to prove Theorem 4,

$$
\begin{aligned}
& \Delta^{\alpha} \geq A \int_{\pi / 4 \geq \theta_{1} \geq 2 \theta_{2} \geq \cdots \geq 2^{k-1} \theta_{k} \geq 0}\left|\prod_{j=1}^{k} \sigma_{N}^{\alpha}\left(\theta_{j}\right)\right|^{2 k} \sin ^{2}\left(\theta_{1} / 2\right) \prod_{j=1}^{k} \sin ^{2}\left(\theta_{j} / 2\right) \\
& \prod_{1 \leq i<j \leq k}\left(\sin ^{2}\left(\left(\theta_{i}-\theta_{j}\right) / 2\right) \sin ^{2}\left(\left(\theta_{i}+\theta_{j}\right) / 2\right)\right) d \theta_{1} \cdots d \theta_{k} \\
& \geq A \int_{\pi / 4 \geq \theta_{1} \geq 2 \theta_{2} \geq \cdots \geq 2^{k-1} \theta_{k} \geq 0} \theta_{1}^{2}\left|\prod_{j=1}^{k} \sigma_{N}^{\alpha}\left(\theta_{j}\right)\right|^{2 k} \theta_{1}^{4 k-2} \theta_{2}^{4 k-6} \cdots \theta_{k}^{2} d \theta_{1} \cdots d \theta_{k} \\
& \geq A \Omega_{1} \Omega_{2},
\end{aligned}
$$

where

$$
\Omega_{1}=\int_{1 / N \geq 2^{\zeta} \theta_{\zeta+1} \geq \cdots 2^{k-1} \theta_{k} \geq 0}\left|\prod_{j=\zeta+1}^{k} \sigma_{N}^{\alpha}\left(\theta_{j}\right)\right|^{2 k} \theta_{\zeta+1}^{4(k-\zeta-1)+2} \cdots \theta_{k}^{2} d \theta_{\zeta+1} \cdots d \theta_{k}
$$

if $1 \leq \zeta \leq k-1$ and $\Omega_{1}=1$ if $\zeta=k$;

$$
\Omega_{2}=\int_{\pi / 4 \geq \theta_{1} \geq \cdots \geq 2^{\zeta-1} \theta_{\zeta} \geq 1 / N}\left|\prod_{j=1}^{\zeta} \sigma_{N}^{\alpha}\left(\theta_{j}\right)\right|^{2 k} \theta_{1}^{4 k} \theta_{2}^{4 k-6} \theta_{\zeta}^{4(k-\zeta)+2} d \theta_{1} \cdots d \theta_{\zeta}
$$

By Lemma 2.8, we know that

$$
\Omega_{1} \geq A N^{2 k(k-\zeta)} \int_{1 / N \geq 2^{\zeta} \theta_{\zeta+1} \geq \cdots \geq 2^{k-1} \theta_{k}} \theta_{\zeta+1}^{4(k-\zeta-1)+2} \cdots \theta_{k-1}^{6} \theta_{k}^{2} d \theta_{1} \cdots d \theta_{k}
$$


Since

$$
\begin{aligned}
2+6+\cdots+4(k-\zeta-1)+\sum_{j=1}^{k-\zeta} 2 & =2(k-\zeta)+4(1+2+\cdots+(k-\zeta-1)) \\
& =2(k-\zeta)+2(k-\zeta)(k-\zeta-1),
\end{aligned}
$$

we easily see that

$$
\Omega_{1} \geq A N^{2 k(k-\zeta)} N^{-(k-\zeta)} N^{-2(k-\zeta)-2(k-\zeta)(k-\zeta-1)}=N^{(k-\zeta)(2 \zeta-1)} .
$$

By (2.9) a simple computation shows that for large $N, \Omega_{2}$ is bounded below by

$$
A N^{-2 \alpha \zeta k} \int_{\pi / 4 \geq \theta_{1} \geq \cdots \geq 2^{\zeta-1} \theta_{\zeta} \geq 1 / N} \theta_{1}^{4 k-(\alpha+1) 2 k} \theta_{2}^{4 k-6-2 k(\alpha+1)} \cdots \theta_{\zeta}^{-\zeta \alpha} d \theta_{1} \cdots d \theta_{\zeta}
$$

Notice that $4 k-(\alpha+1) 2 k>4 k-6-2 k(\alpha+1)>\cdots>4(k-\zeta)+2-2 k(\alpha+1)=$ $-\zeta_{\alpha} \geq-1$. It is easy to see that $\Omega_{2}$ is bounded below by $A N^{-2 \alpha \zeta k}$ if $\zeta_{\alpha}<1$ and by $A N^{-2 \alpha \zeta k} \log N$ if $\zeta_{\alpha}=1$. Using this fact and (5.3), we conclude that

$$
\Delta^{\alpha} \geq A \begin{cases}N^{(k-\zeta)(2 \zeta-1)-2 \alpha \zeta k} & \text { if } \zeta_{\alpha}<1 \\ N^{(k-\zeta)(2 \zeta-1)-2 \alpha \zeta k} \log N & \text { if } \zeta_{\alpha}=1\end{cases}
$$

But using Lemma 2.8 and formula (2.9), one has no difficulty calculating that

$$
I^{\alpha} \leq A \begin{cases}N^{(k-\zeta)(2 \zeta-1)-2 \alpha \zeta k} & \text { if } \zeta_{\alpha}<1, \\ N^{(k-\zeta)(2 \zeta-1)-2 \alpha \zeta k} \log N & \text { if } \zeta_{\alpha}=1\end{cases}
$$

(5.4) and (5.5) furnish the proof of Theorem 2.

\section{Results on $U(n)$ and $\operatorname{USP}(2 n)$}

As we mentioned before, the proofs of the theorems on the groups $U(n)$ and $\mathrm{USP}(2 n)$ are essentially same as those on $\operatorname{SO}(n)$ although we are not able to give a unified proof. In this section, we only list the results obtained in [F3] and [CF], without giving proofs.

On $U(n)$, the Cesàro kernel $K_{N}^{\alpha}(V)$ is defined by (see [G])

$$
\operatorname{det}^{n}\left\{\sum_{k=0}^{N} A_{N-k}^{\alpha-1} B^{k}\left(I-\bar{V}^{, 2 k+1}\right)\right\} /\left\{B_{N}^{\alpha}\left(2 A_{N}^{\alpha}\right)^{n^{2}} \operatorname{det}^{n}\left(I-\bar{V}^{\prime}\right)\right\},
$$

where $B_{N}^{\alpha}$ is the constant such that $\int_{U(n)} K_{N}^{\alpha}(V) d V=1$. 
THEOREM 6.2 [F3]. If $n$ is even then

$$
\lim _{N \rightarrow \infty} \int_{U(n)} K_{N}^{\alpha}(V U) f(V) d V=f(U)
$$

for any continuous function $f$ on $U(n)$ if and only if $\alpha>(n-1) / n$. If $n$ is even then

$$
\lim _{N \rightarrow \infty} \int_{U(n)} K_{N}^{\alpha}(V U) f(V) d V=f(U)
$$

for any continuous function $f$ on $U(n)$ if and only if $\alpha \geq(n-1) / n$.

On USP $(2 n)$, the Cesàro kernel $K_{N}^{\alpha}$ is defined by (see [G])

(6.3) $\operatorname{det}^{n+1 / 2}\left\{\sum_{k=0}^{N} A_{N-k}^{\alpha-1} V^{k}\left(I-\bar{V}^{\prime-2 k+1}\right)\right\} / B_{N}^{\alpha}\left(2 A_{N}^{\alpha}\right)^{n(2 n+1)} \operatorname{det}^{n+1 / 2}\left(I-\bar{V}^{\prime}\right)$

where $B_{N}^{\alpha}$ is the constant such that $\int_{\mathrm{USP}(2 n)} K_{N}^{\alpha}(V) d V=1$.

THEOREM 6.4 [CF]. $\lim _{N \rightarrow \infty} \int_{\mathrm{USP}(2 n)} K_{N}^{\alpha}(V U) f(V)=f(U)$ for any continuous function $f$ on $\operatorname{USP}(2 n)$ if and only if $\alpha>(2 n-2) /(2 n+1)$.

\section{Extensions to compact Lie groups}

From (1.4) and (1.5), we naturally define an analog of the Cesàro kernel on a general compact Lie group $G$ as follows.

Fix a maximal torus $\mathbb{T}$ in $G$ of dimension $l$. Let $\theta=\left(\theta_{1}, \ldots, \theta_{l}\right)$ be a regular coordinate of $\mathbb{T}$. We define the Cesàro kernel on $G$ by

$$
K_{N}^{\alpha, m}(x)=\prod_{j=1}^{l}\left\{\sigma_{N}^{\alpha}\left(\theta_{j}\right)\right\}^{m} / B_{N}^{\alpha, m}, \quad \alpha>-1,
$$

where $m$ is a suitable positive integer,

$$
B_{N}^{\alpha . m}=\int_{G}\left\{\sigma_{N}^{\alpha}\left(\theta_{j}\right)\right\}^{m} d x,
$$

and $x$ is conjugate to $\operatorname{Exp} \theta \in \mathbb{T}$.

By this definition, we easily see that

(a) if $G=U(n)$ and $m=n$, then $K_{N}^{\alpha, n}(x)$ is the Cesàro kernel on $U(n)$ studied in Section 6;

(b) if $G=U(n), m=2 n$ and $a=1$, then $K_{N}^{1,2 n}(x)$ is the Jackson kernel studied in [G, Chapter 4]; 
(c) if $G=\mathrm{SO}(n)$ and $m=n-1$, then $K_{N}^{\alpha, n-1}(x)$ is the Cesàro kernel on $\operatorname{SO}(n)$;

(d) if $G=\operatorname{USP}(2 n)$ and $m=2 n+1$, then $K_{N}^{\alpha, 2 n-1}(x)$ is the Cesàro kernel on USP $(2 n)$ studied in Section 6.

Thus by the theorems obtained in this paper, naturally we will guess that, for a suitable integer $m$, there exists a number $\alpha_{0}$ depending on $m, l$ and $\operatorname{dim}(G)$ such that

(i) if $m$ is even, then $\lim _{N \rightarrow \infty} K_{N}^{\alpha, m} * f(x)=f(x)$ for all continuous function $f$ if and only if $\alpha \geq \alpha_{0}$;

(ii) if $m$ is odd, then $\lim _{N \rightarrow \infty} K_{N}^{\alpha, m} * f(x)=f(x)$ for any continuous function $f$ if and only if $\alpha>\alpha_{0}$.

Here, a suitable integer $m$ means that the critical index $\alpha_{0}$ should be less than 1 . Otherwise (for instance when $\alpha_{0}=1$ ), the Cesàro kernels $K_{N}^{\alpha_{0}, m}$ will identically be positive kernels no matter whether $m$ is odd or even.

We will treat this general case in future publications.

\section{Acknowledgement}

I would like to thank the referee for pointing out this extension of the Cesàro kernel to a general compact Lie group.

\section{References}

[B] H. Boerner, Representations of groups (North-Holland Publishing Co., Amsterdam, 1970).

[C] E. Cartan, 'Sur les domain bornés homogenes de l'espace de n variables', Abh. Math. Sem. Univ. Hamburg 11 (1936), 106-162.

[CF] D. Chen and D. Fan, 'Cesàro kernels on symplectic groups', unpublished manuscript, 1994.

[Cl] J. L. Clerc, 'Sommes de Riesz et multiplicateurs sur un groupe Lie compact', Ann. Inst. Fourier (Grenoble) 24 (1974), 149-172.

[F1] D. Fan, 'Cesàro means of Fourier series on rotation groups', Proc. Amer. Math. Soc. 123 (1995), 1105-1114.

[F2] - 'Salem theorem on compact Lie groups', Chinese Ann. of Math. Ser. A 6 (1985), $397-410$.

[F3] _ 'Cesàro kernels on unitary groups', unpublished manuscript, 1995.

[G] S. Gong, Harmonic analysis on classical groups (Springer, Berlin, 1991).

[GST1] S. Guilini, P. M. Soardi and G. Travaglini, 'A Cohen type inequality for compact Lie groups', Proc. Amer. Math. Soc. 77 (1979), 359-364.

[GST2] _ 'Norms of characters and Fourier series on compact Lie groups', J. Funct. Anal. 46 (1982), 88-101.

[H] L. Hua, Harmonic analysis of functions of several variables in the classical domains, Trans. Math. Monographs 6 (Amer. Math. Soc., Providence, 1963).

[M] C. Meaney, 'Unbounded Lebesgue constants on compact groups', Monatsh. Math. 91 (1981), $119-129$. 
[W] H. Weyl, The classical groups (Princeton Univ. Press, Princeton, 1939).

[Z] A. Zygmund, Trigonometric series, 2nd edition (Cambridge Univ. Press, Cambridge, 1968).

Department of Mathematical Sciences

University of Wisconsin-Milwaukee

Milwaukee, WI 53201

USA

e-mail: fan@alpha1.csd.uwm.edu 